\title{
Parallel solution of multiphase transport problem
}

\author{
G. Kosec \\ Jožef Stefan Institute, Department of Communication Systems, Slovenia
}

\begin{abstract}
In this paper we tackle solidification of a binary alloy that is modelled by fluid flow in free and porous media, heat and mass transport, phase change modelled by eutectic phase diagram, and microscopic species transport. The model is formulated through four tightly coupled Partial Differential Equations describing conservation laws, namely Navier Stokes equation, Darcy equation, and two convective-diffusive equations for describing heat and solute transport. The PDEs are supported by constitutive relations, contributing additional information regarding the diffusion transport, stresses, interfacial forces and buoyancy forces. The solution of the problem at hand is addressed from computational point of view, i.e. the numerical solution procedure is formulated in a way suitable for parallel execution, especially for multi and many core architectures. Results are presented in terms of macrosegregation maps, and parallel efficiency analyses. Keywords: meshless, parallel, fluid flow, solidification of a binary alloy, macrosegregation.
\end{abstract}

\section{Introduction}

Multiphase transport problems govern several interesting problems ranging from global weather systems to specific technological processes. In this paper we focus on the technological process of solidification, which is modeled with models based on thermodynamics and continuum mechanics in solids and fluids simultaneously [1]. The main incitement of the related research is to predict defects that occur during the solidification process, for example solute segregation $[2,3]$, i.e. the spatial variation of material composition within the solidified product that can severely deteriorate the quality of the final product. 
Solidification process is, at least in most practical cases, impossible to treat in a closed form. Therefore, the use of numerical modelling and simulation is the only option to effectively tackle the problem. The main complexities in numerical treatment of the solidification models are moving boundaries, high gradients in governing fields, strong couplings between the transport equations, coupling between different flow regimes, unstable flow of metallic fluids and completely advective transport. Regardless the substantial effort and resources invested to study the behavior of different numerical methods in the prediction of segregation [4-6], the commonly agreed solution is still not available. Although, a macrosegregation maps, i.e. the global segregation throughout the whole domain, can be predicted with a good agreement between different numerical solutions, the segregation on a smaller scale still remain unsolved problem. More details about the numerical simulation of segregation can be found in recent papers $[7,8]$, as well as experimental discussion of the topic in [9].

In the majority of the solidification simulations the Finite Volume Method (FVM), the Finite Difference Method (FDM), or the Finite Element Method (FEM) [10] are used. However, reports on successful application of the meshless methods [11] in the simulation of solidification have been also recently reported $[7,8]$. One of the promising meshless techniques is the Meshless Local Strong Form Method (MLSM) that is essentially a meshless generalization of the FDM. The basic concept MLSM is to approximate considered fields with the nodal trial functions over the local support domain, which is then further manipulated to compute all required differential operations. Such an approach is used in similar methods known as Diffuse Approximate Method (DAM) [12], Local Radial Basis Function Collocation Methods (LRBFCM) [13], Generalized FDM [14], Collocated discrete least squares (CDLS) meshless [15], etc.

Regardless the numerical method, the solution algorithms are executed on computers. To effectively address the problems of the solidification process by simulations, the execution time has to be addresses adequately. The main advantage of the MLSM, from the computation point of view, is that the localization reduces inter-processor communication, which is often a bottleneck of parallel algorithms [16]. In this paper a parallel solution procedure for solving the solidification problem, based on the MLSM numerical technique, is discussed. The discussion is supported with simulation results and execution performance analyses.

\section{Solidification model}

Considered model is also referred as to a "minimal" solidification model, since it is simplified to the largest possible degree, however, still accurate enough to capture several interesting phenomena like macro and meso segregation [7]. The model originates in the paper of $\mathrm{Ni}$ and Beckermann [17] and was later employed in many works [18-20], including in a call for a benchmark solution [6]. The set of governing equations is based on continuum conservation laws and related constitutive relations, it comprises energy and solute transport, incompressible 
Newtonian and porous Darcy fluid flow, and Eutectic phase diagram coupled with the solute transport over the phase front:

Mass conservation:

$$
\nabla \cdot \mathbf{v}=0
$$

Momentum transport:

$$
\rho \frac{\partial \mathbf{v}}{\partial t}+\frac{\rho}{f_{L}}(\nabla \mathbf{v}) \mathbf{v}=
$$

$$
-f_{L} \nabla P+\mu \nabla^{2} \mathbf{v}-f_{L} \frac{\mu}{K} \mathbf{v}+f_{L} \mathbf{b}
$$

Heat transport:

$$
\rho \frac{\partial h}{\partial t}+\rho \mathbf{v} \cdot \nabla h=\lambda \nabla^{2} T
$$

Solute transport:

$$
\frac{\partial C}{\partial t}+\mathbf{v} \cdot \nabla C_{L}=0
$$

Mixture velocity:

$$
\mathbf{v}=f_{L} \mathbf{v}_{L}
$$

Kozeny-Carman relation: $\quad K=K_{0} f_{L}^{3} /\left(1-f_{L}\right)^{2}$,

Boussinesq approximation: $\mathbf{b}=\rho_{\text {ref }}\left[1-\beta_{T}\left(T-T_{\text {ref }}\right)-\beta_{C}\left(C_{L}-C_{\text {ref }}\right)\right] \mathbf{g}$,

Enthalpy formulation: $\quad h=c_{p} T+f_{L} L$,

Mixture concentration: $\quad C=\left[f_{L}+\left(1-f_{L}\right) k_{p}\right] C_{L}$,

Phase diagram:

$$
T=T_{F}+m_{L} C_{L}
$$

In the above model following quantities are used: mixture velocity $\mathbf{v}$, intrinsic liquid velocity $\mathbf{v}_{L}$, enthalpy $h$, average concentration $C$, and the pressure $P$, respectively. The permeability $K$ is defined through a permeability constant $K_{0}$ and the liquid fraction $f_{L}$. The thermo-physical properties, i.e. viscosity $\mu$, thermal conductivity $\lambda$, specific heat $c_{p}$ and density $\rho$ are assumed to be equal and constant in both phases. The buoyancy term $\mathbf{b}$ depends on the temperature $T$, the liquid concentration $C_{L}$, the thermal expansion coefficient $\beta_{T}$, the concentration expansion coefficient $\beta_{C}$, the reference density $\rho_{\text {ref }}$ given at the reference temperature $T_{r e f}$ and the reference concentration $C_{r e f}$. The binary phase diagram is defined by the liquidus slope $m_{L}$, the equilibrium partition coefficient $k_{p}$ and fusion temperature of the pure solvent $T_{F}$. Symbols $t$ and $\mathbf{g}$ stand for time and gravity acceleration, respectively. 


\section{Parallel numerical algorithm}

To solve PDEs at hand a MLSM numerical technique is used. The main goal is to evaluate partial differential operator $L$, in a present case only first and second derivatives, of the considered field $\theta$. In MLSM this is done by applying $L$ on the approximation of a considered field over the local support domain, i.e. only small local sub-set of nodes

$$
L \theta(\mathbf{r})=\sum_{n=1}^{N_{S}}\left(L \chi_{n}(\mathbf{r})\right) \theta\left(\mathbf{r}_{n}\right)
$$

where $\mathbf{r}=(\mathrm{x}, \mathrm{y}), N_{s}$ and $\chi$ stand for position vector, number of support nodes, and shape functions, respectively. To construct shape functions and theirs derivatives one has to: distribute nodes over the domain, find support domains, and construct appropriate approximation functions. These steps can all be done in a pre-process phase, as long as nodal topology does not change during the simulation. Later, in the simulation, only convolution of shape functions and values of considered fields in support domain nodes is required to evaluate a general $L$. More details about the MLSM can be found in [7, 21-24].

The temporal discretization is done by explicit time stepping

$$
\theta^{t+1}(\mathbf{r})=L\left(\theta^{t}(\mathbf{r})\right)
$$

where $t$ denotes time-step index. Since all new time-step values are computed only from the previous ones, the algorithm do not need to communicate with neighbouring nodes to evaluate operator $L$.

The pressure-velocity coupling is performed also locally by Artificial Compressibility Method (ACM) [25].

And finally, consideration of equations (5) to (10) do not require any differentiation of governing fields and therefore it is local by default.

The complete locality of the introduced algorithm has several beneficial effects. Besides simplicity and straightforward implementation, there are high opportunities to fully exploit modern computer architectures through different parallel computing strategies [16, 26]. Figure 1 summarizes the solution procedure, note that tasks that are directly parallelized are marked as "parallel". Note also that those parts of the code present bulk of the execution time.

In this paper the OpenMP (Open Multi-Processing) Application Programming Interface (API) that supports multi-platform shared-memory multiprocessing programming is used for demonstration of the parallel implementation. Since all spatial loops are completely independent no additional effort is required to apply shared-memory parallelization, i.e. each spatial loop is simply executed with pragma omp parallel for with static scheduling. 


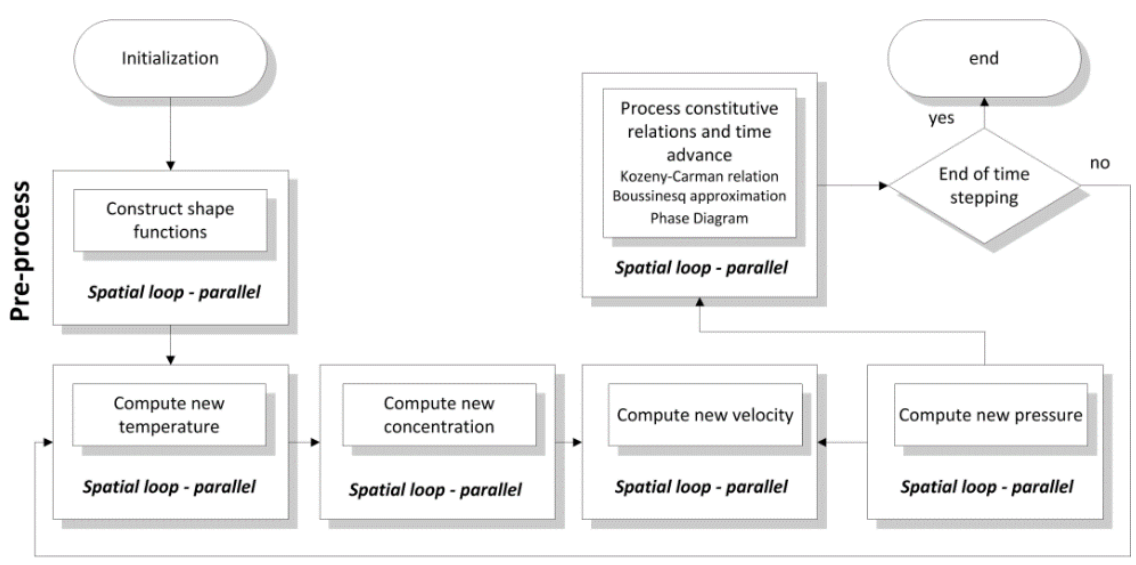

Temporal loop

Figure 1: Diagram of parallel numerical algorithm.

\section{Results}

\subsection{Integration of solidification model}

The numerical integration of the $\mathrm{Sn}-10 \% \mathrm{~Pb}$ solidification is presented in Figure 2 in terms of segregation map, i.e. the contour plot of concentration. The simulation starts with a well-mixed $\left(C_{o}=10 \%\right)$, stationary $(\mathbf{v}=0)$ over-heated $\left(T_{0}=220^{\circ} \mathrm{C}\right)$ liquid. The heat transport is driven by extracting heat from the right side of the domain (10x6 cm rectangular cavity), described by the Robin boundary condition. Since the problem is symmetric, only a half of the domain needs to be numerically computed $(5 \times 6 \mathrm{~cm})$. The symmetry is coped with Neumann boundary conditions applied for all fields on the symmetry line. The domain is thermally isolated, a constant concentration is prescribed and no-slip and no-permeable velocity boundary conditions are applied on all boundaries, except the right one, i.e. the symmetry line. The initially uniformly heated liquid cools from the right side, which induces the thermo-solutal natural convection, i.e. the fluid flow. The main characteristics of the segregation are visible soon after the beginning of the solidification. Note that the solute is transported only by advection, i.e. the segregation characteristics, at least on a global scale, depends only on the flow structures. A positive segregation patch at the bottom of the enclosure and a negative segregation patch in the upper part are soon clearly visible. The channellike anomalies in concentration field occur as a consequence of instabilities in the porous mushy zone. The mechanism behind that phenomenon is still not fully explained, however, it has been observed also experimentally [27], which confirms that such behavior is not a product of numerical instabilities. More details, including all parameters, thermo-physical properties, and boundary conditions can be found in $[7,8]$. 

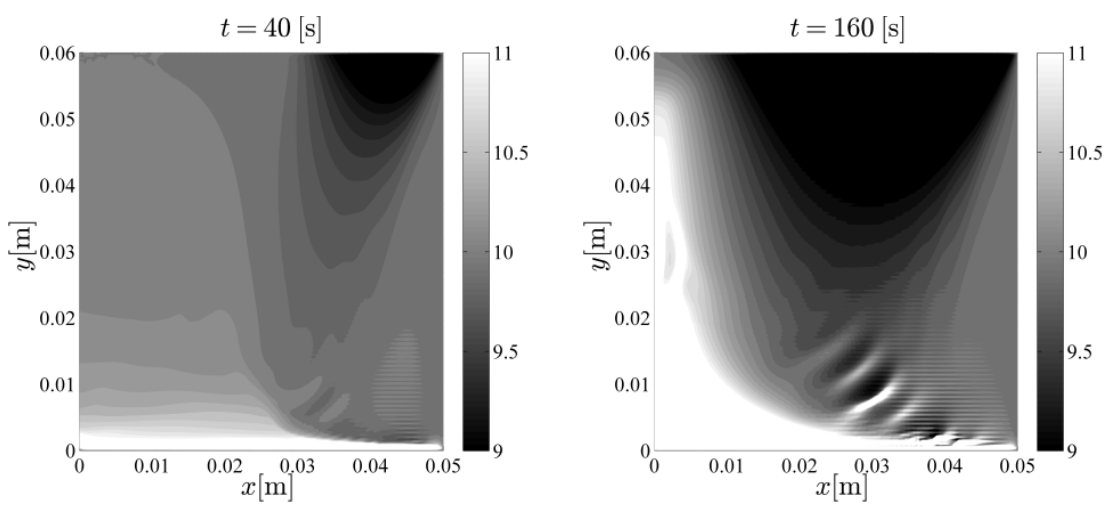

Figure 2: $\quad$ Simulated segregation map after $40 \mathrm{~s}$ (left) and after $160 \mathrm{~s}$ (right).

\subsection{Execution performance}

The performance of the execution is assessed through the speedup measurements, defined as

$$
S=\frac{t_{C}^{1}}{t_{C}^{N_{C}}},
$$

where $t_{C}^{N_{C}}$ stands for the execution time on $N_{C}$ cores. The speedup with respect to the problem size, i.e. the number of computational node is presented, for 2,4 , 8, and 16 cores, in Figure 3. The speedup, as expected, depends on the number of involved cores as well as the problem size. The speedup is governed at least by two effects: available floating point processors and memory access latencies. In cases, when more cores are used, each time a core modifies a memory location on its local cache, it invalidates the caches on other cores that occupy the same memory location. While this frequently happens on shared data, it also happens on private data, because multiple variables might inhabit the same cache block. On the other hand, more cores provide more processing power needed to convolute shape functions and values of considered field in support domain nodes to evaluate differential operations. The interplay of those two effects determines the final speedup. In [23] a detailed analysis of such a phenomena is presented and supported with low-level cache hit rate measurements.

There are also several other factors that have might affect the parallel execution efficiency, e.g. motherboard architecture, bandwidths of data and program buses, cache policies, accumulation of caches, etc.

Tests have been performed with an in-house code written in $\mathrm{C}++$ programming language, compiled with GCC 4.8 compiler with full optimization enabled, on a computer system with four Intel Xeon E7450 processors, each with six cores, system clock of $2.40 \mathrm{GHz}, 1066 \mathrm{MHz}$ front side bus (FSB), $64 \mathrm{~GB}$ of shared main memory and without hyperthreading technology. The system has three levels of cache hierarchy: Each core has L1 execution cache $(32 \mathrm{~KB})$ to store micro- 


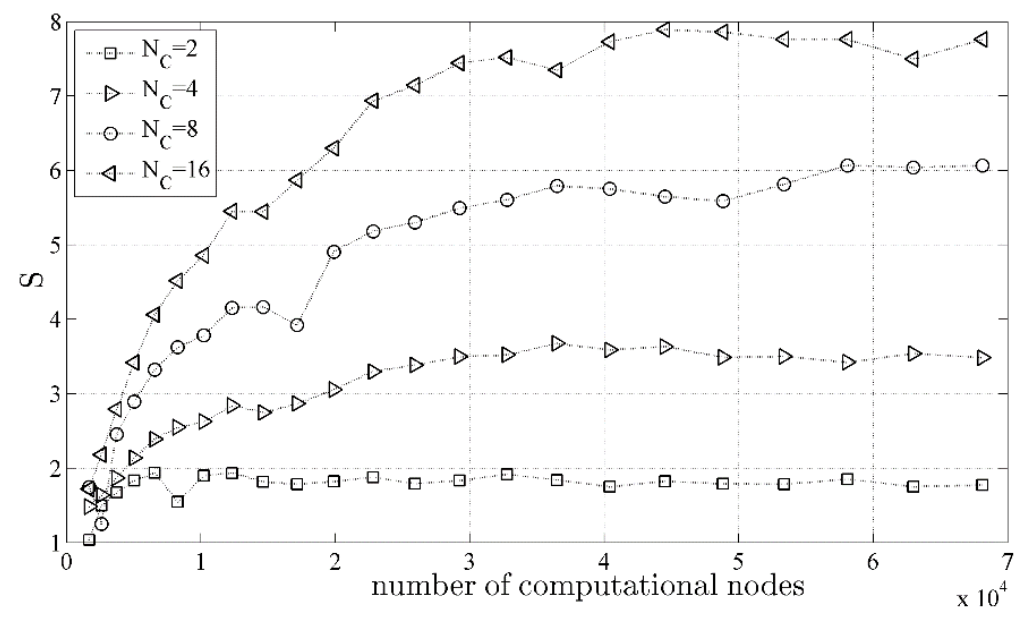

Figure 3: Speedup with respect to the number of involved cores and problem size, i.e. number of computational nodes.

operations (shortest decode time on cache hits) and data cache (32 KB) to improve data tracing. Each pair of cores shares $3 \mathrm{MB}$ of $\mathrm{L} 2$ cache, for a total of $9 \mathrm{MB}$ of shared L2 cache per processor. Each processor has $12 \mathrm{MB}$ of shared L3 cache. The 6-core modules communicate through 64 GB of shared main memory.

\section{Conclusion}

In this paper a local algorithm for solving multiphase problems, namely solidification of a binary alloy, is presented. All elements of the algorithm are formulated in way that only few supporting nodes have to be accessed during the simulation in order to compute required partial derivatives. Besides local spatial discretization, an explicit temporal stepping and ACM are used to assure complete localization of the solution procedure. Consequently, a minor amount of effort and expertise are required to parallelize it with an OpenMP API. Execution performance is demonstrated on an off-the-shelf computer server with 4 CPUs, each with 6 cores. Despite the fact that practically no effort was required to execute the code in parallel, the decent speedups have been observed. The shared memory parallelization efficiency depends on the computer architecture. For example, in $[23,28]$ the super-linear speedup is demonstrated on execution of MLSM in solely thermo-fluid flow simulation. A solidification model, considered in this paper, requires much more numerical attention and therefore more detailed analysis of memory accesses would be required to drawn more detailed conclusion, however, that is out of the scope of this paper.

\section{Acknowledgement}

We acknowledge the financial support from the Slovenian Research Agency under the program group P2-0095. 


\section{References}

[1] Dantzig, J. \& Rappaz, M., Solidification, EPFL Press: Laussane, 2009.

[2] Flemings, M. C \& Nereo, G. E., Macrosegregation, part I. Transactions of Society for Metals AIME, 239, pp. 1449-1461, 1967.

[3] Hebditch, D. J. \& Hunt, J. D., Observations of ingot macrosegregation on model systems. Metallurgical transactions, 5, pp. 1557-1564, 1974.

[4] Combeau, H., Bellet, M., Fautrelle, Y., Gobin, D., Arquis, E., Budenkova, O., Dussoubs, B., Duterrail, Y., Kumar, A., Goyeau, B., Mosba, S, Quatravaux, T, Rady, M. A., Gandin, C. A. \& Založnik, M., A Numerical Benchmark on the Prediction of Macrosegregation in Binary Alloys, Frontiers in Solidification Science, 2011.

[5] Combeau, H., Bellet, M., Fautrelle, Y., Gobin, D., Arquis, E., Budenkova, O., Dussoubs, B., Du Terrail, Y., Kumar, A., Gandin, Ch- A., Goyeau, B., Mosbah, S., Quatravaux, T., Rady, M. \& Založnik, M., Analysis of a numerical benchmark for columnar solidification of binary alloys. IOP Conference Series: Materials Science and Engineering, 33, 2012.

[6] Bellet, M., Combeau, H., Fautrelle, Y., Gobin, D., Rady, M., Arquis, E., Budenkova, O., Dussoubs, B., Duterrail, Y., Kumar, A., Gandin, C. A., Goyeau, B., Mosbah, S. \& Zaloznik, M., Call for contributions to a numerical benchmark problem for 2D columnar solidification of binary alloys. International Journal of Thermal Sciences, 48, pp. 2013-2016, 2009.

[7] Kosec, G. \& Šarler, B., Simulation of macrosegregation with mesosegregates in binary metallic casts by a meshless method. Engineering Analysis with Boundary Elements, 45, pp. 36-44, 2014.

[8] Kosec, G., Založnik, M., Šarler, B. \& Combeau, H., A Meshless Approach Towards Solution of Macrosegregation Phenomena. CMC: Computers, Materials, \& Continua, 22, pp. 1-27, 2011.

[9] Sarazin, J.R. \& Hellawell, A., Channel formation in $\mathrm{Pb}-\mathrm{Sn}, \mathrm{Pb}-\mathrm{Sb}$, and $\mathrm{Pb}-$ $\mathrm{Sn}-\mathrm{Sb}$ alloy ingots and comparison with the system NH4CI-H2O. Metallurgical transactions, 19, pp. 1861-1871, 1988.

[10] Rappaz, M., Bellet, M. \& Deville, M., Numerical Modelling in Materials Science and Engineering, Springer-Verlag: Berlin, 2003.

[11] Atluri, S. N. \& Shen, S., The Meshless Method, Tech Science Press: Encino, 2002.

[12] Wang, C. A., Sadat, H. \& Prax, C., A new meshless approach for three dimensional fluid flow and related heat transfer problems. Computers and Fluids, 69, pp. 136-146, 2012.

[13] Šarler, B., From global to local radial basis function collocation method for transport phenomena. Advances in Meshfree Techniques, pp. 257-282, 2007.

[14] Yang, C., Tang, D., Yuan, C., Kerwin, W., Liu, F., Canton, G., Hatsukami, T. S. \& Atluri, S., Meshless generalized finite difference method and human carotid atherosclerotic plaque progression simulation using multi-year MRI patient-tracking data. CMES: Computer Modeling in Engineering \& Sciences, 28, pp. 95-107, 2008. 
[15] Arzani, H. \& Afshar, M. H., Solving Poisson's equation by the discrete least square meshless method. WIT Transactions on Modelling and Simulation 42, pp. 23-31, 2006.

[16] Trobec, R., Šterk, M. \& Robič, B., Computational complexity and parallelization of the meshless local Petrov-Galerkin method. Computers \& Structures, 87, pp. 81-90, 2009.

[17] Ni, J. \& Beckermann, C., A volume-averaged two-phase model for transport phenomena during solidification. Metallurgical and Materials Transactions, 22B, pp. 349-361, 1991.

[18] Drew, D. A., Mathematical modeling of two-phase flow. Annual Review of Fluid Mechanics, 15, pp. 261-291, 1983.

[19] Wang, C. Y. \& Beckermann, C., Equiaxed dendritic solidification with convection: Part I. multiscale/multiphase modeling. Metallurgical and Materials Transactions, A27, pp. 2754-2764, 1996.

[20] Goyeau, B., Bousquet-Melou, P., Gobin, D., Quintard, M. \& Fichot, F., Macroscopic modeling of columnar dendritic solidification. Computational \& Applied Mathematics, 23, pp. 381-400, 2004.

[21] Kosec, G. \& Zinterhof, P., Local strong form meshless method on multiple Graphics Processing Units. CMES: Computer Modeling in Engineering \& Sciences, 91, pp. 377-396, 2013.

[22] Kosec, G. \& Trobec, R., Simulation of semiconductor devices with a local numerical approach. Engineering Analysis with Boundary Elements, 50, pp. 69-75, 2015.

[23] Kosec, G., Depolli, M., Rashkovska, A. \& Trobec, R., Super linear speedup in a local parallel meshless solution of thermo-fluid problems. Computers \& Structures, 133, pp. 30-38, 2014.

[24] Kosec, G., Meshless Solution of Incompressible Flows in Complex Domains, Proceedings of the Ninth International Conference on Engineering Computational Technology, Civil-Comp Press, 2014.

[25] Arpino, Fausto, Massarotti, Nicola, Mauro, Alessandro \& Nithiarasu, Perumal, Artificial compressibility based CBS solutions for double diffusive natural convection in cavities. International Journal of Numerical Methods for Heat \& Fluid Flow, 23, pp. 205-225, 2013.

[26] Bollig, E. F., Flyer, N. \& Erlebacher, G., Solution to PDEs using radial basis function finite-differences (RBF-FD) on multiple GPUs. Journal of Computational Physics 231, pp. 7133-7151, 2012.

[27] Asai, S., Sahara, T. \& Muchi, I., Theoretical analysis and model study of the formation of A-type segregation in ingots. Tetsu-To-Hagane/Journal of the Iron and Steel Institute of Japan, 63, pp. 1512-1519, 1977.

[28] Kosec, G., Trobec, R., Depolli, M. \& Rashkovska, A., Multicore parallelization of a meshless PDE solver with OpenMP, ParNum 11, 2011. 